Three sets of electrodiagnostic criteria for establishing primary demyelination in chronic polyneuropathy are evaluated. Sensitivity is assessed in 70 patients with clinically established chronic inflammatory demyelinating polyradiculoneuropathy (CIDP). The criteria use different abnormal values, one adjusts for the effects of axonal loss, while another relies only on conduction velocity. However, even when consideration is given to sufficient number of nerves tested, there is no significant difference $(P=0.37)$ in diagnostic sensitivity among them, with $48 \%$ to $64 \%$ of CIDP patients fulfilling criteria for primary demyelination. Specificity is assessed by applying the criteria to 47 patients with motor neuron disease and 63 patients with diabetic polyneuropathy. No patients meet any of the criteria. Further analysis shows that as sensitivity increases specificity decreases, because of overlapping distributions of nerve conduction abnormalities in these neuropathic disorders. A sensitivity of approximately $66 \%$ is a practical limit for electrodiagnostic criteria in CIDP.

Key words: demyelination - polyneuropathy • electrodiagnosis $\bullet$ sensitivity MUSCLE \& NERVE 14:968-976 1991

\title{
COMPARISON OF ELECTRODIAGNOSTIC CRITERIA FOR PRIMARY DEMYELINATION IN CHRONIC POLYNEUROPATHY
}

\author{
MARK B. BROMBERG, MD, PhD
}

The diagnosis of chronic inflammatory demyelinating polyradiculoneuropathy (CIDP) is made from the clinical history, physical examination, and laboratory tests. ${ }^{11}$ Measurement of nerve conduction is helpful because it permits electrodiagnostic assessment of demyelination, the primary underlying pathology. ${ }^{11,20}$ Expected changes are slowed conduction velocity, abnormal temporal dispersion, and conduction block. ${ }^{14}$ The wide range of normal values for these measures make it important to establish abnormal values which are reasonably predictive of primary demyelination. In early studies of CIDP, slowed motor nerve conduction velocity and subjective estimates of abnormal temporal dispersion were emphasized. ${ }^{4,11,16,20}$

From the Department of Neurology, University of Michigan Medical Center, Ann Arbor, Michigan.

Acknowledgments: The author thanks Dr. James W. Albers for advice and comments, Dr. Judith Bromberg for advice on statistics, and Dr. Ivo Drury for reviewing the manuscript.

Presented in part at the Thirty-Seventh Annual Meeting of the American Association of Electrodiagnostic Medicine, Chicago, September 1990.

Address reprint requests to Dr. M.B. Bromberg, Department of Neurology, University of Michigan Medical Center, Taubman Center 1920/0316 1500 East Medical Center Drive, Ann Arbor, MI 48109-0316.

Accepted for publication September 15, 1990.

CCC 0148-639X/91/0100968-09\$04.00

(c) 1991 John Wiley \& Sons, Inc.
In later studies, formal sets of criteria were proposed to establish electrodiagnostic evidence of primary demyelination which consider distal latency, conduction velocity, partial conduction block or abnormal temporal dispersion, and F-wave latency.

Three sets of criteria have been recently proposed for electrodiagnostic identification of patients with CIDP. ${ }^{1,3,4}$ They differ in several respects; they use different values to define abnormalities, 1 attempts to account for the effect of axonal loss on nerve conduction, while another considers only conduction velocity (Table 1 ). The sensitivity of these criteria for identifying CIDP has not been evaluated in a large group of patients, nor have the criteria been compared with each other. Furthermore, specificity (true negative rate) in identifying primary demyelination has not been established in groups of patients with neuropathic disorders in which demyelination is unexpected or is considered to be of secondary importance to axonal damage. ${ }^{6}$

In this report, three sets of criteria are evaluated for sensitivity using nerve conduction data from patients with the clinical diagnosis of CIDP. Specificity is tested on nerve conduction data from patients with motor neuron disease (MND) and diabetic polyneuropathy (DP). Factors in the mea- 
Table 1. Proposed electrodiagnostic criteria for primary demyelination in CIDP.

Set $A^{3}$

Must demonstrate 3 of the following abnormalities in motor nerves.

1. Reduced conduction velocity in 2 or more nerves:

$<75 \%$ of LLN. ${ }^{*}$

2. Partial conduction block or abnormal temporal dispersion in 1 or more nerves: $<70 \%$ P/D ratio $\dagger$

3. Prolonged distal latency in 2 or more nerves:

$>130 \%$ of ULN.

4. Prolonged $F$-wave latency in 1 or more nerves:

$>130 \%$ of ULN.

Modified A

Same as above, except that reduction in conduction velocity and prolonged distal latency need be present in only 1 nerve to be counted as 1 of the 3 abnormalities.

Set $B^{1}$

Must demonstrate 3 of the following abnormalities in motor nerves.

1. Reduction in conduction velocity in 2 or more nerves:

$<80 \%$ of LLN if CMAP amplitude $>80 \%$ of LLN, or

$<70 \%$ of LLN if CMAP amplitude $<80 \%$ of LLN.

2. Partial conduction block or abnormal temporal dispersion in 1 or more nerves (median, ulnar, peroneal):

a. partial conduction block:

$<80 \%$ P/D ratio if duration of negative peak of proximal CMAP is $<115 \%$ of distal CMAP duration.

b. abnormal temporal dispersion and possible conduction block:

$<80 \%$ P/D ratio if duration of negative peak of proximal CMAP is $>115 \%$ of distal CMAP duration.

3. Prolonged distal latency in 2 or more nerves:

$>125 \%$ of ULN if CMAP amplitude $>80 \%$ of LLN, or

$>150 \%$ of ULN if CMAP amplitude $<80 \%$ of LLN.

4. Prolonged F-wave latency in 2 or more nerves:

$>120 \%$ of ULN if CMAP amplitude is $>80 \%$ LLN, or

$>150 \%$ of ULN if CMAP amplitude is $<80 \%$ LLN, or absent $F$ wave after 10 to 15 trials.

Set $C^{3}$

Must demonstrate the following abnormality in motor nerves.

1. Reduction in conduction velocity in 2 or more nerves:

$<70 \%$ of LLN.

Modified C

Same as above, except that reduction in conduction velocity need be present in only 1 nerve to fulfill criteria.

*LLN: lower limit of normal

TPID: proximal to distal compound muscle action potential (CMAP) amplitude ratio.

$\mp U L N$ : upper limit of normal.

surement of nerve conduction which influence sensitivity are identified, and evidence is presented for an upper limit of sensitivity which preserves reasonable specificity.

\section{METHODS}

Nerve conduction data were obtained from 3 groups of patients. (a) Seventy patients, who were actively followed in our clinic, fulfilled clinical criteria for idiopathic CIDP. ${ }^{11}$ Criteria included progressive, relapsing or stepwise course with weakness and sensory disturbance, cerebrospinal fluid cytoalbuminic dissociation in most patients, response to corticosteroids and therapeutic plasma exchange, and exclusion of other diagnoses, such as associated monoclonal gammopathy, ${ }^{13}$ multifo- cal conduction block neuropathy, ${ }^{19}$ or systemic dysimmune disorders. (b) Forty-seven patients, who were actively followed in our clinic, fulfilled criteria for MND. ${ }^{18}$ These included clinical evidence for upper motor neuron loss, electrodiagnostic evidence for lower motor neuron loss in at least 3 extremities, and exclusion of other diagnoses. Data reviewed for patients with CIDP and MND were obtained at the time of their initial evaluation. (c) Sixty-three patients, referred to the EMG Laboratory, had insulin-dependent or noninsulin-dependent diabetes mellitus and clinically evident polyneuropathy of mild-to-moderate severity. This group represented consecutive, unselected patients referred for confirmation of the polyneuropathy.

Nerve conduction studies were performed us- 

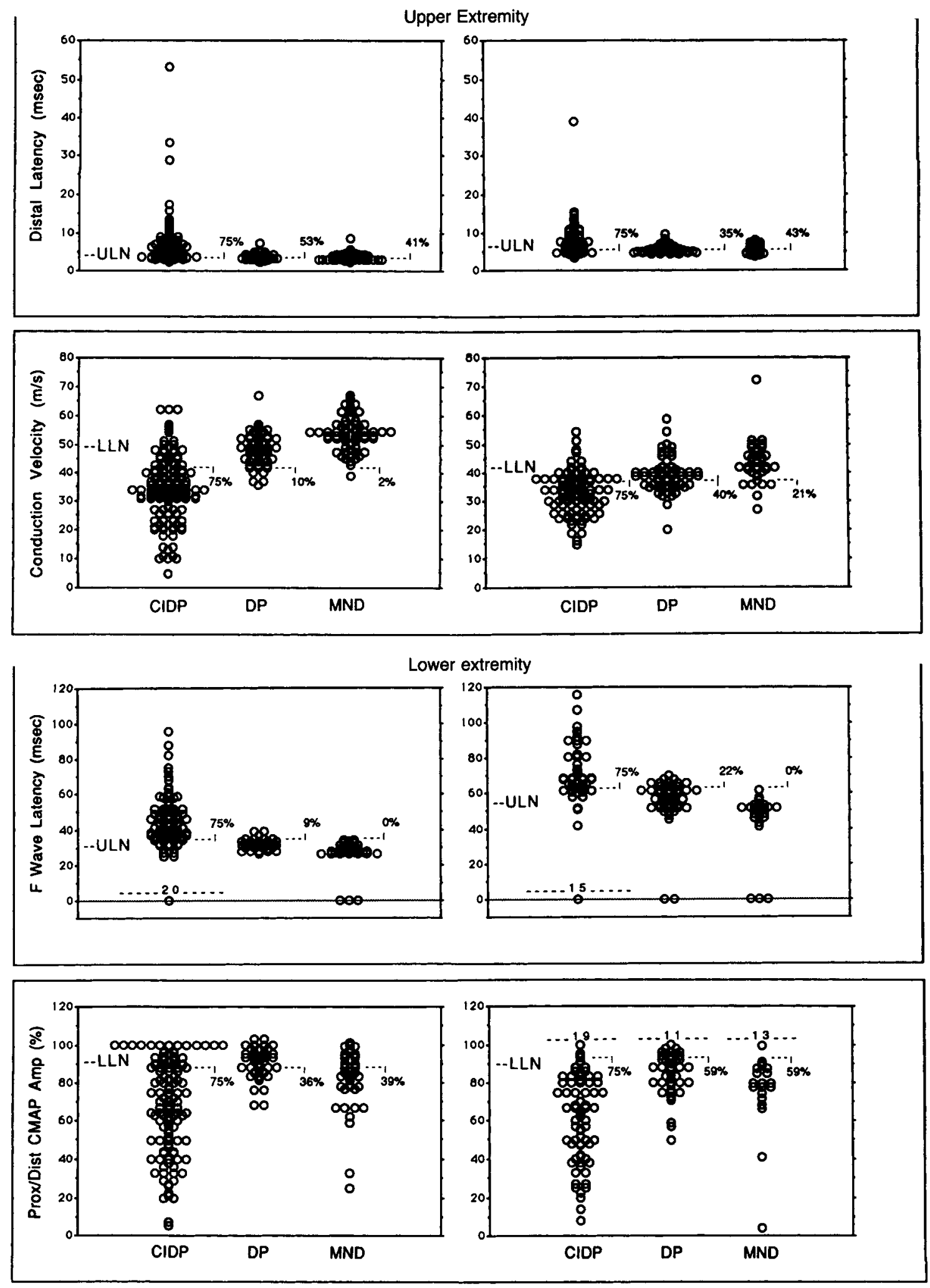

FIGURE 1. Distribution of values for motor nerve conduction measures. Values for upper extremity nerve displayed in left scattergrams, values for lower extremity in right scattergrams. Horizontal lines at left hand side mark upper and lower limits of normal values (ULN and LLN, respectively). Horizontal lines to right of scattergrams mark values for 75 th percentile for patients with CIDP (true positive) and percentile values (false positive) for patients with diabetic polyneuropathy (DP) and motor neuron disease (MND). Dashed lines with numbers at $0 \%$ and $100 \%$ indicate coincident values. 
ing conventional techniques. ${ }^{2}$ These included recording of the compound muscle action potential (CMAP) with surface disc electrodes. Proximal stimulation sites for the ulnar and peroneal nerves were below the elbow and fibular head, respectively. Skin temperature was maintained between $32^{\circ}$ and $34^{\circ} \mathrm{C}$ with hot packs. When bilateral measurements were made on specific nerves, only measures from one side of the body were analyzed (the side with the greatest number of nerve studied).

Nerve conduction values were reviewed to determine if they exceeded abnormal limits listed in Table 1 for 3 sets of criteria, designated sets $A^{3}$, $B^{1}$, and $C^{4}$. A modification was made to the second criterion of set $\mathrm{B}$. Because duration measurements of the negative component of the CMAP were not available for every patient, no distinction was made between partial conduction block and abnormal temporal dispersion with possible conduction block; the term "conduction block/abnormal temporal dispersion" used in this report refers to measurements in which the proximal: distal CMAP ratio was lower than the limiting values listed in criteria sets A and B.

The number of abnormal nerve conduction values for each patient was tabulated to determine if the patient completely fulfilled requirements for primary demyelination. Abnormal values for individual measures were calculated as percentages of laboratory upper limits of normal (ULN) or lower limits of normal (LLN) values. The limits of normal were set at the range for values not normally distributed (tibial motor nerve distal latency and conduction velocity) or at $2 \mathrm{SD}$ above or below normal mean values for normally distributed measures. The normal laboratory values were obtained from subjects of both genders, and whose ages ranged from the first to the seventh decades. The ULN and LLN are marked in Figure 1.

Chi-square tests for differences in proportions

Table 2. Demographic features of 3 groups of patients.

\begin{tabular}{|c|c|c|c|}
\hline & CIDP & $\begin{array}{c}\text { Motor } \\
\text { neuron } \\
\text { disease }\end{array}$ & $\begin{array}{c}\text { Diabetic } \\
\text { polyneuropathy }\end{array}$ \\
\hline \multicolumn{4}{|l|}{ Gender: } \\
\hline Men & $41(59 \%)$ & $26(55 \%)$ & $28(44 \%)$ \\
\hline Women & $29(41 \%)$ & $21(45 \%)$ & $35(56 \%)$ \\
\hline \multicolumn{4}{|c|}{ Age at time of study: } \\
\hline Mean (years) & 48 & 62 & 55 \\
\hline Range (years) & $4-76$ & $19-82$ & $22-84$ \\
\hline
\end{tabular}

were performed. Because of multiple comparisons, $P$-values less than .01 are considered statistically significant, $P$-values between .01 and .05 marginally significant, and $P$-values greater than .05 not significant.

\section{RESULTS}

The age and gender distributions (Table 2) are similar to those reported for CIDP and MND patients. $^{4,18}$

Sensitivity of Criteria A, B, and C for CIDP. Sensitivities (true positive rate) of each of the 3 criteria were determined using available nerve conduction data for each patient. Sets A, B, and C had sensitivities of $50 \%, 46 \%$, and $43 \%$, respectively, and differences in these rates were not statistically significant $(P=.69)$ (Table 3). Not every testable motor nerve was evaluated in each patient so that failure to fulfill a set could possibly result from insufficient sampling. Exclusion of such patients and recalculation of sensitivity resulted in $64 \%$ of patients fulfilling criteria for set A, $60 \%$ for set B, and $48 \%$ for set $\mathrm{C}$. The difference in proportions were not statistically significant $(P=.37)$. The apparent increase in sensitivity may be an overestimate because not every untested nerve would be abnormal.

Comparison of the 3 Criteria. The three criteria differ from each other by using different abnormal values for individual measures of nerve conduction and by requiring different numbers of abnormal measures to meet full criteria (Table 1). The sensitivity of each set was studied by reviewing the data to determine (a) which individual nerve conduction measure was most frequently abnormal, and (b) which measure was most often abnormal in the required number of nerves (Table 4). For example, when the abnormal level for conduction velocity was $75 \%$ of the LLN, as in set A (Table 1$)$, significantly more $(P=.0001)$ individual nerves were abnormal than when the level was $70 \%$ of the LLN as in set C (Table 4). Similar findings were noted for F-wave latency, and significantly more $(P=.0001)$ nerves were abnormal in set B (120\% of ULN) compared with A (130\% of ULN). In set B, F-wave latency was most frequently judged abnormal at $120 \%$ of the ULN because most nerves had a CMAP amplitude greater than $80 \%$ of the LLN (Table 1).

When the data were reviewed to see whether abnormalities occurred in 1 or 2 nerves, as re- 
Table 3. Sensitivity of 3 sets of electrodiagnostic criteria for primary demyelination for patients with the clinical diagnosis of CIDP, and specificity for patients with motor neuron disease and diabetic polyneuropathy.

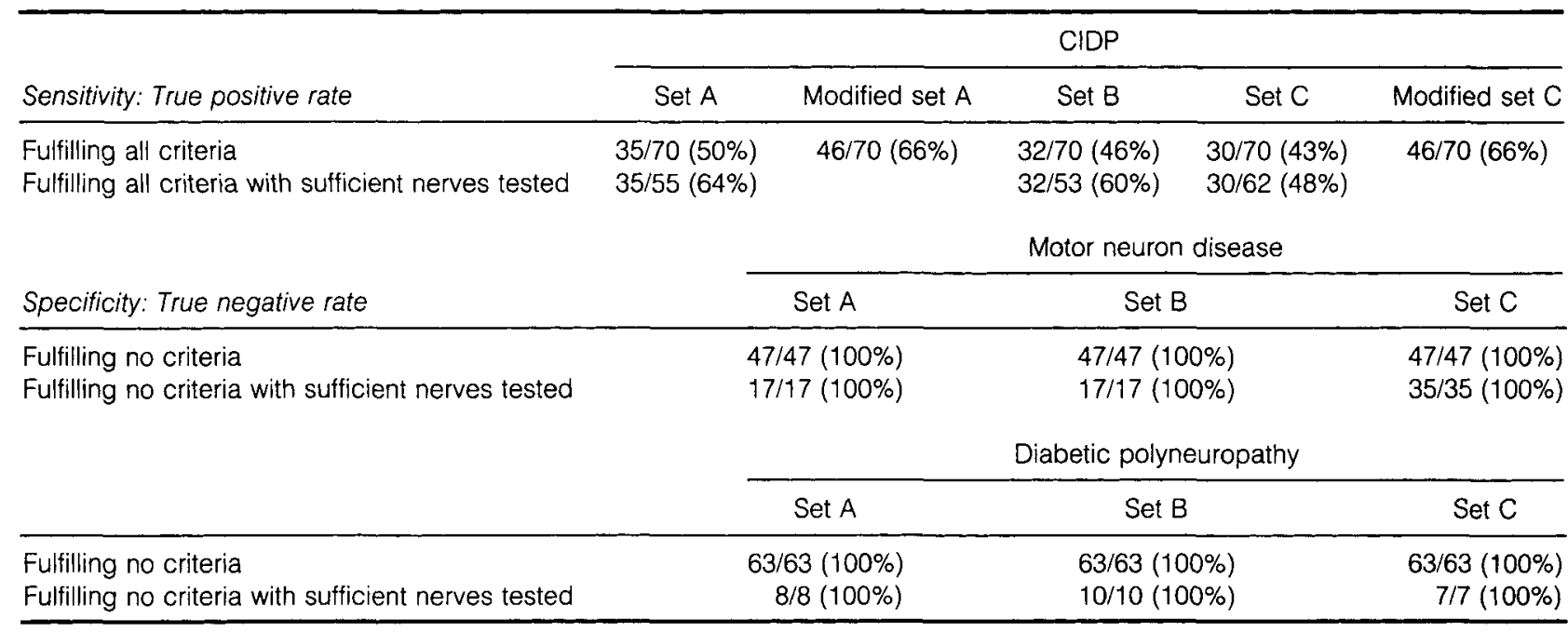

Table 4. Number of abnormalities in all motor nerves tested according to three sets of criteria for electrodiagnostic evidence for primary demyelination in patients with CIDP, motor neuron disease, and diabetic polyneuropathy.

\begin{tabular}{lcc}
\hline & & CIDP \\
\cline { 2 - 3 } & Set A & Set B \\
\hline Conduction velocity & $153 / 216(71 \%)$ & $110 / 216(51 \%)$ \\
Distal latency & $89 / 227(39 \%)$ & $82 / 227(36 \%)$ \\
Conduction block/abnormal dispersion & $98 / 210(47 \%)$ & $120 / 210(57 \%)$ \\
F-wave latency & $66 / 172(38 \%)$ & $111 / 172(65 \%)$ \\
& & Motor neuron disease \\
\cline { 2 - 3 } & Set A & Set B \\
\hline Conduction velocity & $0 / 109(0 \%)$ & $2 / 109(2 \%)$ \\
Distal latency & $2 / 95(2 \%)$ & $1 / 95(1 \%)$ \\
Conduction block/abnormal dispersion & $12 / 95(13 \%)$ & $22 / 95(23 \%)$ \\
F-wave latency & $0 / 59(0 \%)$ & $6 / 65(9 \%)$ \\
\hline & & Diabetic polyneuropathy \\
\hline & Set A & Set B \\
\hline Conduction velocity & $3 / 105(3 \%)$ & $3 / 105(3 \%)$ \\
Distal latency & $4 / 156(3 \%)$ & $3 / 156(2 \%)$ \\
Conduction block/abnormal dispersion & $6 / 103(6 \%)$ & $8 / 91(9 \%)$ \\
F-wave latency & $0 / 89(0 \%)$ & $1 / 103(17 \%)$ \\
\hline
\end{tabular}

quired by each set (Table 1), the sensitivity of individual measures changed (Table 5). For example, conduction block/abnormal temporal dispersion was the most commonly fulfilled measure, in part because only 1 nerve was required to be abnormal in both sets A and B. On the other hand, although $F$ waves were frequently abnormal in individual nerves in set $\mathbf{B}$, this abnormality was less com- monly fulfilled because it was required in 2 nerves.

Specificity of Criteria A, B, and C in Other Neuropathic Disorders. Specificity (true negative rate) of the 3 sets was tested with nerve conduction data from patients with MND and DP, and no patients fulfill any set for primary demyelination (Table 3). 


\begin{tabular}{|c|c|c|c|c|c|}
\hline $\begin{array}{l}\text { Percentage of } \\
\text { ULN or LLN }\end{array}$ & $\begin{array}{l}\text { CMAP } \\
\text { amplitude }\end{array}$ & $\begin{array}{l}\text { Distal } \\
\text { latency }\end{array}$ & $\begin{array}{l}\text { Conduction } \\
\text { velocity }\end{array}$ & $\begin{array}{l}\text { F-wave } \\
\text { latency }\end{array}$ & $\begin{array}{l}\text { Proximal/ } \\
\text { distal ratio }\end{array}$ \\
\hline 100 & LLN & ULN & LLN & ULN & LLN \\
\hline 80 & B & & B-1 & & B-1 and 2 \\
\hline 75 & & & A & & \\
\hline 70 & & & $\mathrm{~B}-2$ and $\mathrm{C}$ & & $A$ \\
\hline 120 & & & & B-1 & \\
\hline 125 & & B-1 & & & \\
\hline 130 & & $A$ & & A & \\
\hline 150 & & B-2 & & B-2 & \\
\hline
\end{tabular}

Fewer nerves were tested than in patients with CIDP, but when consideration was given for sufficient numbers tested, only 1 patient with DP could possibly fulfill set C.

Several differences in nerve conduction abnormalities were noted between primary demyelinating and primary axonal disorders (Table 4). First, abnormalities of conduction velocity, distal latency, and F-wave latency were uncommon in both MND and DP, but occurred frequently in CIDP. Second, conduction block/abnormal temporal dispersion was the most common abnormal measure in MND and DP, but occurs significantly less frequently in MND and DP than in CIDP ( $P$ $<.0001$ for each comparison).

Analysis of Different Criteria. Sensitivity of electrodiagnostic criteria can be increased by 3 methods. First, the required number of abnormal conduction measures can be reduced. This method was used in set $C$ (Table 1), but sensitivity was only $43 \%$ when abnormal conduction velocity was required in 2 nerves (Table 3). When abnormal conduction velocity was required in only $l$ nerve (modified set C, Table 1), sensitivity increased to $66 \%$ (Table 3 ).

Second, all nerve conduction measures can be evaluated, but each given equal weight so that an abnormal measure in 1 nerve can be used to fulfill criterion. Using the abnormal values from set $\mathrm{A}$, but modified, so that conduction velocity and distal latency criteria were fulfilled when abnormal in only 1 nerve (modified set $A$, Table 1 ), sensitivity increased to $66 \%$, while specificity was unchanged at $100 \%$ (Table 3 ).

Third, abnormal values for each nerve conduction measure can be made less stringent, with a goal of achieving at least $75 \%$ sensitivity and $75 \%$ specificity for each measure. The distribution of nerve conduction values for each conduction measure revealed substantial overlap among the 3 disorders and with the normal range (Fig. 1). Abnormal values, which include $75 \%$ of CIDP patients, were near the ULN and LLN for each measure, thereby potentially including normal subjects.

The values marking the 75 th percentile for sensitivity for CIDP patients were also values which include up to $59 \%$ of patients with MND and DP. When values at the 75th percentile for individual nerve conduction measures were substituted for the values in sets $\mathrm{A}$ and $\mathrm{B}$, overall sensitivity for CIDP patients rose to nearly $100 \%$, but specificity fell to nearly $0 \%$. This increase in sensitivity and decrease in specificity was due to the fact that multiple nerves, each with values at $75 \%$ sensitivity and $75 \%$ specificity, were tested in an individual patient. Although many combinations can be chosen of abnormal values, and numbers of abnormal measures required to fulfill all criteria, $66 \%$ sensitivity appeared to be a practical limit attainable without reducing specificity. This level of sensitivity can be achieved by requiring only a single abnormality in 1 nerve (modified set $\mathrm{C}$ ), or less stringent abnormalities in several nerves (modified set $\mathrm{A}$ ).

\section{Discussion}

Evaluation of 3 electrodiagnostic criteria for establishing the diagnosis of demyelinating polyneuropathy indicates that sets A and B have similar sensitivity. The greater complexity of set $B$, based on the amplitude of the distal CMAP, does not appear to offer an advantage. This is found to occur for several reasons. The distal CMAP amplitude is below $80 \%$ of the LLN in $38 \%$ of tested nerves, 
Table 6. Number of patients fulfilling individual nerve conduction criterions for electrodiagnostic evidence for primary demyelination.

\begin{tabular}{lll}
\hline & \multicolumn{1}{c}{ CIDP } \\
\cline { 2 - 3 } & Set A & Set B \\
\hline Conduction velocity (2 nerves) & $37 / 70(53 \%)$ & $38 / 70(54 \%)$ \\
Distal latency (2 nerves) & $29 / 70(41 \%)$ & $27 / 70(39 \%)$ \\
Conduction block/abnormal dispersion (1 nerve) & $59 / 70(84 \%)$ & $64 / 70(91 \%)$ \\
F-wave latency (set A 1 nerve, set B 2 nerves) & $38 / 70(54 \%)$ & $34 / 70(49 \%)$
\end{tabular}

\begin{tabular}{lcc} 
& & Motor neuron disease \\
\cline { 2 - 3 } & Set A & Set B \\
\hline Conduction velocity (2 nerves) & $0 / 47(0 \%)$ & $0 / 47(0 \%)$ \\
Distal latency (2 nerves) & $0 / 47(0 \%)$ & $0 / 47(0 \%)$ \\
Conduction block/abnormal dispersion (1 nerve) & $11 / 47(23 \%)$ & $17 / 47(36 \%)$ \\
F-wave latency (set A 1 nerve, set B 2 nerves) & $0 / 47(0 \%)$ & $0 / 47(0 \%)$ \\
& & Diabetic polyneuropathy \\
\hline Conduction velocity (2 nerves) & Set A & Set B \\
Distal latency (2 nerves) & $0 / 63(0 \%)$ & $1 / 63(2 \%)$ \\
Conduction block/abnormal dispersion (1 nerve) & $0 / 63(0 \%)$ & $0 / 63(0 \%)$ \\
F-wave latency (set A 1 nerve, set B 2 nerves) & $6 / 63(10 \%)$ & $0 / 63(25 \%)$ \\
\hline
\end{tabular}

and for the majority of nerves, therefore, the abnormal values in set $B$ are less stringent than those in set $\mathrm{A}$ in this group of CIDP patients (Table 5). However, the 2 sets of values are sufficiently similar to include nearly equal percentages of patients for each measure (Table 6). Set $B$ requires abnormal $\mathrm{F}$ waves in 2 nerves, but permits the absence of an $F$ wave after a suitable number of trials to count toward fulfillment (Table 1). Although set A does not count an absent $\mathrm{F}$ wave as abnormal, it requires only one abnormal response, which results in no significant differences in the proportion of patients fulfilling this measure in sets $\mathrm{A}$ and $\mathrm{B}$.

Increasing the sensitivity of the criteria proved difficult. While it is possible to calculate abnormal values which achieve $75 \%$ sensitivity for individual measures for CIDP patients, these values overlap conduction values from normal subjects and patients with primary axonal loss (Fig. 1). Substitution of these calculated abnormal values in sets $\mathrm{A}$ and $B$ results in nearly $100 \%$ sensitivity, but much lower specificity with false inclusion of nearly $100 \%$ of patients with MND and DP.

Although the 3 criteria require a specific number of abnormal measures to achieve fulfillment, none addresses the question of how many motor nerves should be tested. There is a practical limit to the number of motor nerves accessible for test- ing, commonly the ulnar, median, peroneal, and tibial nerves. Bilateral measures of nerve conduction from the same nerve are highly correlated in normal subjects and in CIDP patients (Bromberg, unpublished observations). Accordingly, to insure independent assessments, it is prudent to include data for a particular nerve from only 1 side of the body.

When nerves are unresponsive to electrical stimulation, they do not contribute to any criteria, but only $15 \%$ of nerves tested are unresponsive in this sample of CIDP patients. Although a number of patients fulfill criteria for primary demyelination when only 2 nerves are tested, it is reasonable to require testing of 4 motor nerve before concluding that a patient does not meet the criteria, with notation of the number of unresponsive nerves. In the same vein, an $\mathrm{F}$-wave response should be considered absent only after a suitable number of trials, as required in set $\mathrm{B}$.

It is difficult to compare the $48 \%$ to $66 \%$ sensitivity obtained in this sample of CIDP patients to other samples. Early studies relied on slowed conduction velocity only, and conduction data are frequently expressed in terms of group means. ${ }^{11,20}$ In one series, only $67 \%$ of CIDP patients had slowed conduction velocity in a single motor nerve, ${ }^{4}$ and, in the current study group, $66 \%$ of patients fulfill this minimal requirement. 
There are several reasons why sensitivity is not higher. Patients received their initial nerve conduction study at different stages in the course of their disease. ${ }^{11}$ The extent of demyelination and remyelination during the course of the polyneuropathy affects nerve conduction. In acute inflammatory demyelinating polyradiculoneuropathy (AIDP), which has a monophasic time course of demyelination, up to $86 \%$ of patients fulfill a similar set of electrodiagnostic criteria at the peak of symptoms, but only $63 \%$ to $67 \%$ fulfill criteria during the early convalescent phase. ${ }^{2}$ This is a similar percentage to that seen in CIDP using the AIDP criteria. ${ }^{5}$ Another factor is that the only commonly available measure of conduction over proximal nerve segments, where demyelination may be severe, is $\mathrm{F}$ wave latency. ${ }^{11}$

Partial conduction block/abnormal temporal dispersion is a sensitive measure for detecting primary demyelination, and may occur to a greater degree in more proximal nerve segments. ${ }^{7,17} \mathrm{Re}$ cording responses over more proximal segments may be one method of increasing the likelihood of detecting this abnormality. This can be performed practically along the ulnar nerve by stimulating below and above the elbow, at the mid-arm, at the axilla, and at Erb's point. ${ }^{7}$

There are no false positive errors for the three criteria. DP was chosen as a difficult test of the criteria. Although pathological changes support axonal degeneration as the primary process and demyelination as a lesser process, ${ }^{6}$ electrodiagnostic features of DP include reduced CMAP amplitude, prolonged distal and $\mathrm{F}$-wave latencies, and slowed conduction velocity. ${ }^{10}$ Specificity has also been demonstrated by the diagnosis of CIDP superimposed on DP when nerve conduction studies fulfill criteria for primary demyelination. ${ }^{8}$ All diabetic patients had symptomatic polyneuropathy of mild to moderate degree; all but 2 had abnormal spontaneous activity on needle electromyography, and many had absent evoked motor and sensory nerve responses.

MND was chosen because it is a motor neuronopathy with reduced CMAP, mildly prolonged distal latency, and slowed conduction velocity, presumably secondary to loss of large motor fibers. ${ }^{9}$ The only abnormal measure encountered is conduction block/abnormal temporal dispersion. Apparent conduction block can occur when there are few motor fibers remaining, and the motor units are enlarged; in this situation, normal temporal dispersion can reduce phase addition in the re- sponse recorded after stimulation at proximal sites. $^{7}$ Ten of 12 nerves showing block in patients with MND have low CMAP amplitude, and likely demonstrate apparent conduction block by this mechanism. Attention to conduction block is important because true conduction block may be the first diagnostic clue to a special form of multifocal demyelinating motor neuropathy which mimics clinical features of MND, but which is potentially treatable. ${ }^{15,19}$

In summary, the criteria currently proposed for demonstrating electrodiagnostic evidence for primary demyelination, while highly specific, are only moderately sensitive. With few changes in these criteria, the maximal sensitivity with adequate specificity appears to be about $66 \%$. This can be achieved by requiring a single abnormal measure (conduction velocity) in 1 nerve (modified set $\mathrm{C}$ ), or by requiring several different abnormal conduction measures (modified set A). There are arguments favoring use of criteria, which require greater numbers of abnormalities. First, they increase diagnostic confidence by excluding forms of hereditary neuropathies and focal entrapment neuropathies. Hereditary motor sensory neuropathy (type I) would commonly satisfy individual measures of distal latency, conduction velocity, and F-wave latency, but not abnormal conduction block/abnormal temporal dispersion. ${ }^{12,14} \mathrm{~A}$ focal entrapment neuropathy could cause slow nerve conduction. Second, criteria requiring greater numbers of nerve abnormalities permit more detailed analysis of the effects of therapy. For example, reduction of conduction block or abnormal temporal dispersion, and improvement in distal latency, may occur before improvement in conduction velocity during therapy, ${ }^{21}$ and this would be missed if only the latter were being monitored.

Third, nerve conduction studies, as routinely applied to distal limb segments, may underestimate the extent of the demyelinating process. ${ }^{11}$ Since conduction block/abnormal temporal dispersion is a frequently abnormal measure, more extensive testing for this abnormality may increase sensitivity.

Although nerve conduction abnormalities in CIDP appear to be distinct from those in neuropathic disorders without primary demyelination, the correct diagnosis of CIDP must rely upon assessment of all clinical data, and use of a full set of clinical and laboratory inclusion criteria, such as recently proposed ${ }^{1,4}$ will be helpful. 


\section{REFERENCES}

1. Criteria for diagnosis of chronic inflammatory dernyelinating polyneuropathy (CIDP). Report from an Ad Hoc Subcommittee of the American Academy of Neurology AIDS Task Force. Neurology 1991;41:617-618.

2. Albers JW, Donofrio PD, McGonagle TK: Sequential electrodiagnostic abnormalities in acute inflammatory demyelinating polyradiculoneuropathy. Muscle Nerve 1985;8:528539.

3. Albers JW, Kelly JJ: Acquired inflammatory demyelinating polyneuropathies: Clinical and electrodiagnostic features. Muscle Nerve 1989;12:435-451.

4. Barohn RJ, Kissel JT, Warmolts JR, Mendell JR: Chronic inflammatory demyelinating polyradiculoneuropathy. Arch Neurol 1989;46:878-884.

5. Bromberg MB, Albers JW: Nerve conduction studies in chronic and acute inflammatory demyelinating polyradiculoneuropathies. Muscle Nerve 1988;11:960.

6. Brown MJ, Asbury AK: Diabetic neuropathy. Ann Neurol $1984 ; 15: 2-12$.

7. Brown WF, Feasby TE: Conduction block and denervation in Guillain-Barré polyneuropathy. Brain 1984;107:219239.

8. Cornblath DR, Drachman DB, Griffin JW: Demyelinating motor neuropathy in patients with diabetic polyneuropathy. Ann Neurol 1987;22:126.

9. Denys EH: Motor nerve conduction velocities, in Mulder DW (ed): The Diagnosis and Treatment of Amyotrophic Lateral Sclerosis, Boston, Houghton-Mifflin, 1980, pp 105-117.

10. Daube JR: Electrophysiologic testing in diabetic neuropathy, in Dyck PJ, Thomas PK, Asbury AK, Winegrad AI, Porte D (eds): Diabetic Neuropathy. Philadelphia, Saunders, 1987, pp 162-176.
11. Dyck PJ, Lais AC, Ohta M, Bastron JA, Okazaki $H$, Groover RV: Chronic inflammatory polyradiculoneuropathy. Mayo Clin Proc 1975;50:621-637.

12. Dyck PJ, Lambert EH: Lower motor and primary sensory neuron diseases with peroneal muscular atrophy. Arch Neurol 1968;18:603-617.

13. Kelly JJ: The electrodiagnostic findings in peripheral neuropathy associated with monoclonal gammopathy. Muscle Nerve 1983;6:504-509.

14. Lewis RA, Sumner AJ: The electrodiagnostic distinctions between chronic familial and acquired demyelinative neuropathies. Neurology 1982;32:592-596.

15. Lewis RA, Sumner AJ, Brown MJ, Asbury AK: Multifocal demyelinating neuropathy with persistent conduction block. Neurology 1982;32:958-964.

16. McCombe PA, Pollard JD, McLeod JG: Chronic inflammatory demyelinating polyradiculoneuropathy. Brain 1987;110:1617-1630.

17. Mills KR, Murray NMF: Proximal conduction block in early Guillain-Barré syndrome. Lancet 1985;659.

18. Mulder DW: The clinical syndrome of amyotrophic lateral sclerosis. Mayo Clin Proc 1957;32:427-436.

19. Pestronk A, Cornblath DR, Ilyas AA, Baba H, Quarles RH, Griffin JW, Alderson K, Adams RN: A treatable multifocal motor neuropathy with antibodies to GMl ganglioside. Ann Neurol 1988;24:73-78.

20. Prineas JW, McLeod JG: Chronic relapsing polyneuritis. $J$ Neurol Sci 1976;27:427-458.

21. Van der Meche, FG, Vermeulen M, Busch HFM: Chronic inflammatory demyelinating polyneuropathy. Brain $1989 ; 112: 1563-1571$. 